\title{
Semi in-situ measurement of zincate ion concentration near zinc anode using background-oriented Schlieren technique
}

\author{
Yasumasa Ito $\odot,{ }^{1},{ }^{*}$ Xiao Liang, ${ }^{1}$ Kohei Ishikawa, ${ }^{2}$ Toru Ujihara, ${ }^{2}$ Yasuhiko Sakai,${ }^{1}$ and Koji Iwano $\odot^{1}$ \\ ${ }^{1}$ Department of Mechanical Systems Engineering, Nagoya University, Nagoya 464-8603, Japan \\ ${ }^{2}$ Institute of Materials and Systems for Sustainability, Nagoya University, Nagoya 464-8603, Japan
}

(Received 15 August 2019; published 10 December 2019)

\begin{abstract}
In metal-anode batteries such as zinc, the concentration of metal ions in the electrolyte usually plays a significant role in the metal electrodeposition upon charging. This study aims to clarify the concentration of zincate ions near the zinc anode. After preliminary experiments in a minichannel were conducted to identify the system parameters, galvanostatic charging experiments in a zinc battery cell were conducted at current densities of 20 and $40 \mathrm{~mA} \mathrm{~cm}^{-2}$. The bulk concentrations of zincate ions were set to 0.49 and $0.98 \mathrm{~mol} \mathrm{~L}^{-1}$. Images near the zinc anode were captured using an optical microscope to calculate the zincate ion concentration distribution through the background-oriented Schlieren (BOS) technique. They were taken at an equal time intervals to investigate the transition of the concentration diffusion layer. It was confirmed that the BOS technique enables us to quantitatively measure the zincate ion concentration in zinc anode batteries. The results are consistent with the theoretical expectation. The applied current density basically determines the concentration gradient near the anode surface, although it takes time to reach a steady state. The spatial variance in the zincate ion concentration at the anode surface increases with the distance from equilibrium.
\end{abstract}

DOI: 10.1103/PhysRevResearch.1.033162

\section{INTRODUCTION}

\section{A. Background}

A short cycle life from an internal short circuit is a primary issue to be solved in realizing long-life metal-anode rechargeable batteries, such as lithium and zinc batteries [1]. This issue is caused by a nonuniform or dendritic formation of electrodeposited metal upon charging [1-4]. Thus, it is of great importance to understand the electrodeposition mechanism and develop methods that realize a uniform, flat, and dense morphology.

Certain factors influence the metal electrodeposition. The current density is the most direct factor. From a mass transport perspective, the concentration of metal ions in an electrolyte is significant $[5,6]$. Furthermore, the spatial distributions of the current density and metal ion concentration on the electrode surface are as important as their values because their nonuniformity can cause a nonuniform electrodeposition. By contrast, from a perspective of crystal growth, the surface energy and crystallographic orientation strongly affect the electrodeposition, particularly in terms of the morphology [2,7-9]. An overpotential is also an important factor; however, considering that it represents the required driving force $[2,5,7,11]$, which involves all of the above factors, it can

\footnotetext{
*Corresponding author: yito@nagoya-u.jp

Published by the American Physical Society under the terms of the Creative Commons Attribution 4.0 International license. Further distribution of this work must maintain attribution to the author(s) and the published article's title, journal citation, and DOI.
}

be regarded as a measure of the distance from equilibrium $[3,4,10]$.

Among the factors above, the present study focuses on the concentration distribution of metal ions in the electrolyte. Upon charging, whereas the metal ions in the electrolyte are deposited on the anode, the concentration at the anode surface becomes lower than the bulk concentration and a concentration diffusion layer forms. As the concentration decreases, the distance from the equilibrium increases, meaning that a nonuniform deposition becomes more likely to occur. In this respect, particularly in zinc-anode batteries, an electrolyte flow is known to be useful for controlling the electrodeposition $[3,4,12]$ and overpotential [13]. Such a flow brings the system closer to equilibrium by changing the driving force of the ion migration from molecular diffusion to convection, i.e., by supplying a bulk electrolyte with rich ions to the anode surface and thinning the concentration layer. In addition, a classical model calculation indicates that the relationship among the concentration diffusion layer thickness, surface roughness of the deposition or the curvature radius of a dendrite tip, and the height of the deposition determines the following morphology $[14,15]$.

These facts already indicate the significance of elucidating the concentration field of zincate ions in zinc batteries in fundamental studies, but it is also important to know the concentration distribution from a perspective closer to battery research and development. For example, when a dendritic growth appeared together with a nonuniform distribution of zincate ions, it suggests that a control of the concentration field would help to make the morphology flat and dense. As another example, there is a case that an additive changes zinc morphology but the physical mechanism is unknown. If the 
change is attributed to the modification of the concentration field, we become able to show the evidence. Besides, this can lead to the optimizations of the amount and component of the additive.

Riede et al. [16] carried out a numerical simulation to clarify the relationship between the zincate ion concentration within the vicinity of the zinc anode and the zinc morphology. Their results indicate that dendritic deposition starts to appear when the zincate ion concentration on the anode surface reaches a critical concentration (approximately $0.11 \mathrm{~mol} \mathrm{~L}^{-1}$ in their study), which was derived from a molecular diffusion equation. Dundáleka et al. [17] conducted charging experiments on a zinc anode in the flowing electrolyte and investigated the transition of the zinc morphology from an equilibrium perspective. They concluded that the hydrogen bubbles may cause an extra convection within the diffusion layer, which can lead to an enhanced mass transport of zincate ions to the electrode surface. In their case, the surface concentration of the zincate ions is estimated based on a semiempirical mass transfer model.

By contrast, a direct experimental measurement of the concentration is essentially desired. However, to the best of our knowledge, an in situ quantitative measurement of the concentration distribution during battery cycling is extremely challenging and limited [19]. In this respect, we extended the background-oriented Schlieren (BOS) technique [20-23], which was originally developed to visualize a shockwave and calculate the pressure field in air during studies on fluid dynamics. As its name suggests, this technique is a type of Schlieren method, and the spatial density (technically, the refractive index) gradient of the medium is detected by comparing the images with and without disturbances in density. In metal-anode batteries, the electrolyte density changes in accordance with the decrease in the metal ion concentration near the anode upon cycling. This indicates that although the object size and medium are completely different from the original values, the BOS technique can be applied to a battery system. In addition to allowing semi in-situ measurements to be conducted, a large advantage of the BOS technique is its relatively simple and inexpensive experiment setup.

\section{B. Purpose}

The purpose of this study is to quantitatively measure the zincate ion concentration near the zinc anode upon charging and investigate the effects of the current density and bulk concentration of zincate ions, which significantly change the distance from equilibrium. Two sets of experiments were conducted. The first set is preliminary experiments conducted in a minichannel, which were carried out for two objectives. The first objective is to evaluate the reliability and applicability of the BOS technique for an extremely small target using a microscope. The second objective is to identify the system parameter required to calculate the concentration of zincate ions in the following battery experiments. The second set of experiments, which make up the main part of this study, are galvanostatic charge experiments, for which we used zinc as the anode material.

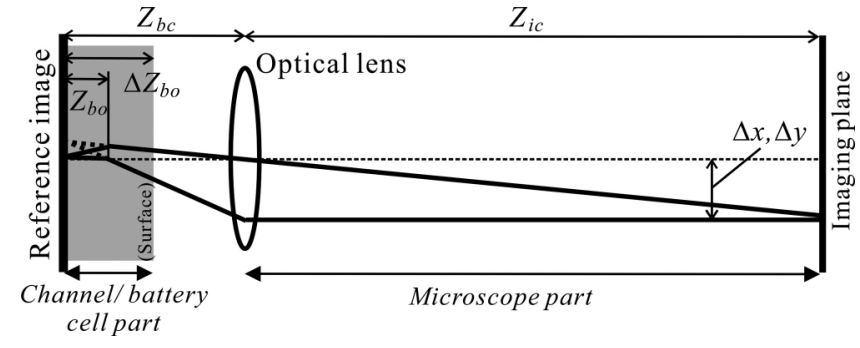

FIG. 1. Schematic of the optical configuration.

\section{BOS TECHNIQUE}

In this section, a brief description of the procedure used for calculating the concentration is shown along with the basics of the BOS technique.

Step 1. Figure 1 shows a schematic of the optical configuration. Using a microscope, reference images consisting of randomly patterned dots placed at the bottom of the minichannel and battery are captured under both nondisturbance and disturbance conditions. More specifically, for the minichannel experiments, the former image is taken when the channel is filled with a stream of sugar water with a uniform concentration, whereas the latter image is taken when two streams of sugar water with different concentrations are introduced. For the battery experiments, the former and latter images are taken before and during charging, respectively.

Step 2. Comparing the images, the latter images show partial distortion owing to the density disturbances, i.e., movement of the dots as compared with the former image. The displacement of each dot has a linear relationship with the spatial density gradient:

$$
\begin{aligned}
& \frac{\partial \rho(x, y)}{\partial x}=n_{0} \frac{\Delta x Z_{b c}}{G Z_{b o} \Delta Z_{b o} Z_{i c}}, \\
& \frac{\partial \rho(x, y)}{\partial y}=n_{0} \frac{\Delta y Z_{b c}}{G Z_{b o} \Delta Z_{b o} Z_{i c}},
\end{aligned}
$$

where $x$ and $y$ are the streamwise and spanwise directions, respectively, $\rho$ is the fluid density, $n_{0}$ is the refractive index of the medium without density disturbances, $\Delta x$ and $\Delta y$ are the displacements of the dots, and $G$ is the Gladstone-Dale coefficient defined by $G=\frac{n-1}{\rho}$. In addition, and $Z_{b o}, \Delta Z_{b o}$, $Z_{i c}$, and $Z_{b c}$ are the distance between the density disturbances and the reference image (technically the half of the channel and battery height in the present cases), the thickness of density disturbances (the channel and battery height), the distance between the lens to the imaging plane, and the distance between the lens and a reference image, respectively. If the system configurations are consistent, Eq. (1) can be rewritten as follows:

$$
\frac{\partial \rho(x, y)}{\partial x}=\frac{n_{0} \mathrm{C}_{\text {sys }}}{G} \Delta x, \quad \frac{\partial \rho(x, y)}{\partial y}=\frac{n_{0} \mathrm{C}_{\text {sys }}}{G} \Delta y,
$$

where $\mathrm{C}_{\mathrm{sys}}$ is a constant summarizing the system parameters such as $Z_{b c}, Z_{b o}, \Delta Z_{b o}$, and $Z_{i c}$.

For the calculation of the dot displacements, a direct cross-correlation method was employed. Note that, to increase the accuracy, the captured image was divided into some 
interrogation areas, and the average displacement was obtained in each area.

Step 3. Here, $\rho(x, y)$ satisfies the following Poisson-type equation;

$$
\frac{\partial^{2}}{\partial x^{2}} \rho(x, y)+\frac{\partial^{2}}{\partial y^{2}} \rho(x, y)=S(x, y),
$$

where $S(x, y)$ is the source term. Because $\frac{\partial \rho}{\partial x}$ and $\frac{\partial \rho}{\partial y}$ are known from step 2, the term on the left can be written as follows using the finite volume method with a second-order central difference scheme:

$$
\begin{aligned}
S(x, y)= & \frac{\partial^{2}}{\partial x^{2}} \rho(x, y)+\frac{\partial^{2}}{\partial y^{2}} \rho(x, y) \\
= & {\left[\frac{\partial \rho(x+\Delta x, y)}{\partial x}-\frac{\partial \rho(x-\Delta x, y)}{\partial x}\right] / 2 \Delta x } \\
& +\left[\frac{\partial \rho(x, y+\Delta y)}{\partial y}-\frac{\partial \rho(x, y-\Delta y)}{\partial y}\right] / 2 \Delta y .
\end{aligned}
$$

Simultaneously, the term on left in Eq. (4) can also be written as follows:

$$
\begin{aligned}
& \frac{\partial^{2}}{\partial x^{2}} \rho(x, y)+\frac{\partial^{2}}{\partial y^{2}} \rho(x, y) \\
& \quad=\frac{\rho(x+\Delta x, y)+\rho(x-\Delta x, y)-2 \rho(x, y)}{\Delta x^{2}} \\
& \quad+\frac{\rho(x, y+\Delta y)+\rho(x, y-\Delta y)-2 \rho(x, y)}{\Delta y^{2}} .
\end{aligned}
$$

From these equations, $\rho(x, y)$ is expressed as

$$
\begin{aligned}
\rho(x, y)= & {\left[\frac{\rho(x+\Delta x, y)+\rho(x-\Delta x, y)}{\Delta x^{2}}\right.} \\
& +\frac{\rho(x, y+\Delta y)+\rho(x, y-\Delta y)}{\Delta y^{2}} \\
& -S(x, y)] /\left(\frac{2}{\Delta x^{2}}+\frac{2}{\Delta y^{2}}\right) .
\end{aligned}
$$

Step 4. The initial $\rho(x, y)$ is set to the bulk concentration at all points and is assigned to the right term of Eq. (6), and the new $\rho(x, y)$, which is different from the bulk concentration, is calculated. The same is applied using the new $\rho(x, y)$ iteratively. The difference between the new and old $\rho(x, y)$ normally declines to a small value. When the summation of the differences at all points becomes smaller than the determined value, we consider the calculation to have converged, i.e., the true $\rho(x, y)$ is obtained at all points.

Regarding the boundary conditions, the Neumann condition with the first-order upwind differential scheme is applied for all boundaries including the solid-liquid interface. The interface is determined based on the brightness value at each pixel.

Step 5. The calculated $\rho(x, y)$ is converted into the concentration of the chemical species (sugar or zincate ions) using the calibration curve between the medium density and the concentration, which is obtained through other preliminary experiments.

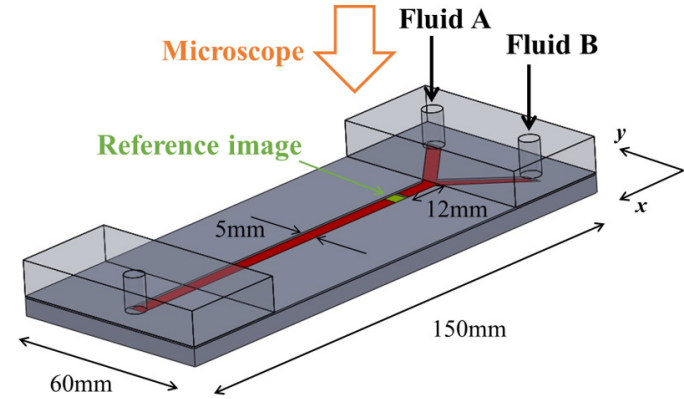

FIG. 2. Schematic of the minichannel.

\section{MINICHANNEL EXPERIMENTS}

\section{A. Experimental apparatus}

Figure 2 shows a schematic of the minichannel. The minichannel is made of an acrylic resin and has a $\mathrm{Y}$ shape with an angle of $30 \mathrm{deg}$ for each inlet. The origin of the coordinate is the root of the Y shape, and the depth $(z)$, width $(y)$, and streamwise $(x)$ lengths of the straight part of the minichannel are $0.5,5$, and $100 \mathrm{~mm}$, respectively. The large aspect ratio of the cross section ensures the twodimensionality of the flow. A reference image is embedded under the channel at $x=12-17 \mathrm{~mm}$ and $y=-6-6 \mathrm{~mm}$ as a part of the channel wall. In addition, to minimize the influence of refraction from the upper wall of the channel, the top wall of the imaging area is replaced with a transparent film with a thickness of $0.1 \mathrm{~mm}$ (Innovect 5735-s). Two syringe pumps (YMCYSP-101) were used to supply streams of sugar water with different concentrations (fluids $\mathrm{A}$ and $\mathrm{B}$ ) into the channel separately. The average cross-sectional velocity at the measurement point was set to $2.0 \mathrm{~mm} \mathrm{~s}^{-1}$. In this case, the Reynolds number based on the hydraulic equivalent diameter and the average cross-sectional flow velocity is 3.6 , meaning the flow is laminar. Therefore, the mixing of the two fluids gradually proceeds only through molecular diffusion at the center of the channel.



FIG. 3. Dot pattern of the reference image. 


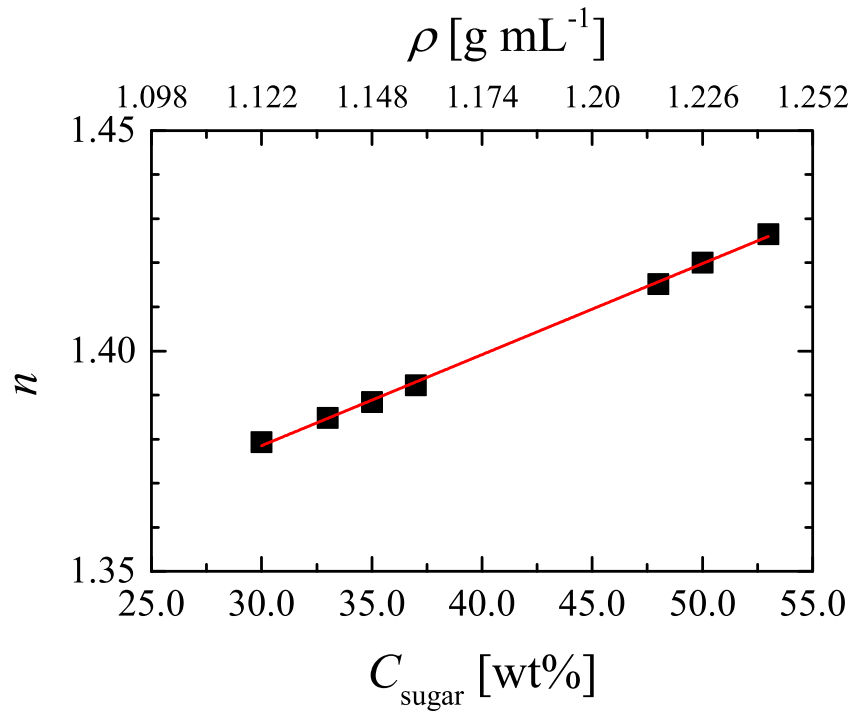

FIG. 4. Relationship between the refraction index against the concentration and the density of the sugar water.

A part of the reference image is shown in Fig. 3. Numerous dots were patterned using chromium etching on a silicon wafer coated with a silicon dioxide film for chemical resistibility. The size of the dots is typically $3 \mu \mathrm{m}$, and the dots are randomly placed with a coverage area of $35 \%$. The thickness of each printed dot is less than $500 \mathrm{~nm}$.

The image was captured using a microscope (Micro Advance AS-500C) from the top of the channel. The spatial resolution is $2592 \times 1944$ pixels. In the present experiments, the magnification of the microscope was set to $\times 200$ and the imaging area was $1.47 \times 1.10 \mathrm{~mm}$. The displacement vectors were obtained using a direct correlation method with an interrogation area of $32 \times 32$ pixels, resulting in a spatial resolution of $18 \mu \mathrm{m}$.

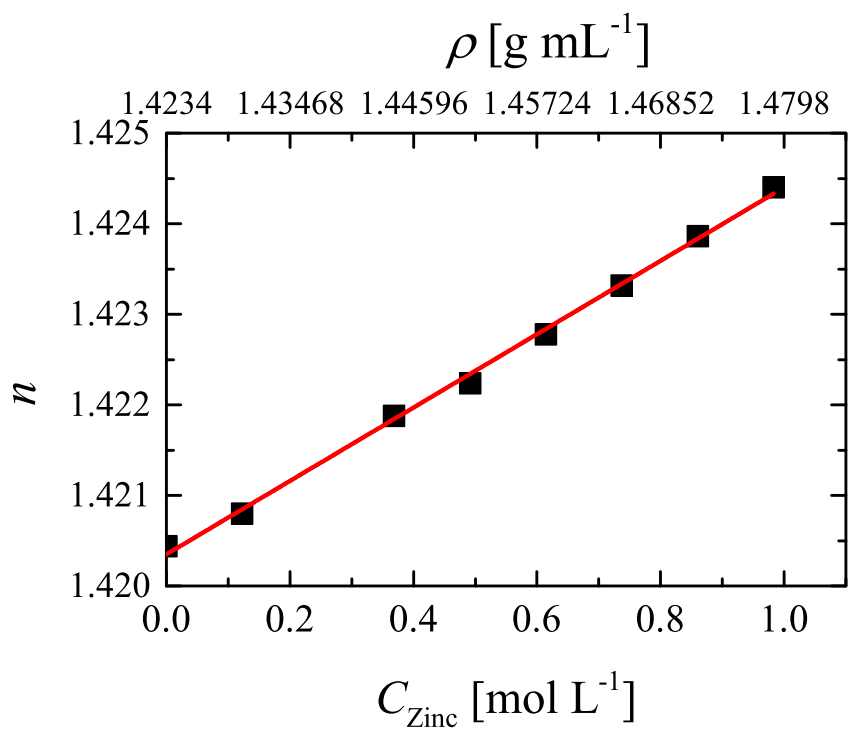

FIG. 5. Relationship between the refraction index against the concentration and the density of the electrolyte with different zincate ion concentrations.
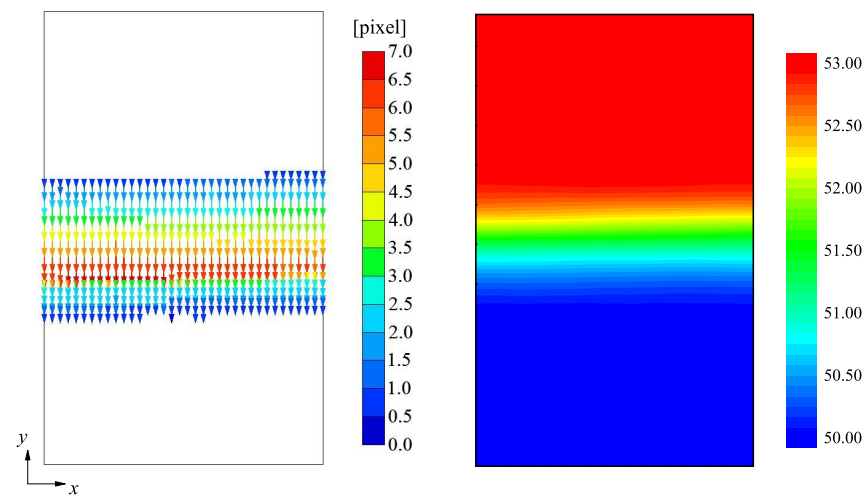

FIG. 6. Example of displacement vector map (left) and color maps of the concentration distribution (right) for 53.0-50.0 wt\% mixing.

\section{B. Experiment condition}

Figure 4 shows the refractive index, $n$, against the concentration $C$ and density $\rho$ of the sugar water. Figure 5 shows the same image but for a $10.0 \mathrm{~mol} \mathrm{~L}^{-1}$ potassium hydroxide $(\mathrm{KOH})$ solution with various zincate ion concentrations, which were used for the main experiments. The refractive index was measured using a refractometer (Atago MASTER-53 $\alpha$ ). It can be confirmed from both figures that a linear relationship exists between the refractive index and the concentration (density). In addition, a comparison of the two figures indicates that the range of the refractive index of the sugar water with a concentration of approximately 50 $53 \mathrm{wt} \%$ covers that for the electrolytes anticipated during the main experiments. This does not necessarily match the range of the refractive index between the two experiments when identifying the system parameters, although to be certain, we set the concentration of fluid A to $53.0 \mathrm{wt} \%$ and that of fluid $\mathrm{B}$ to 50.0 and $51 \mathrm{wt} \%$ in the present experiments.

\section{Results}

Figures 6 and 7 show examples of a displacement vector map (left) and color maps of the concentration distribution (right) for 53.0-50.0 wt\% mixing and 53.0-51.0 wt $\%$ mixing,
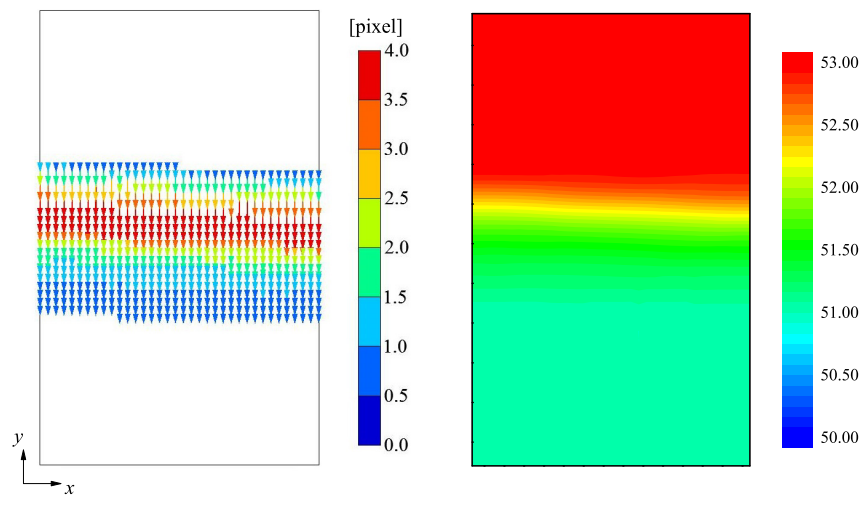

FIG. 7. Example of displacement vector map (left) and color maps of the concentration distribution (right) for $53.0-51.0 \mathrm{wt} \%$ mixing. 


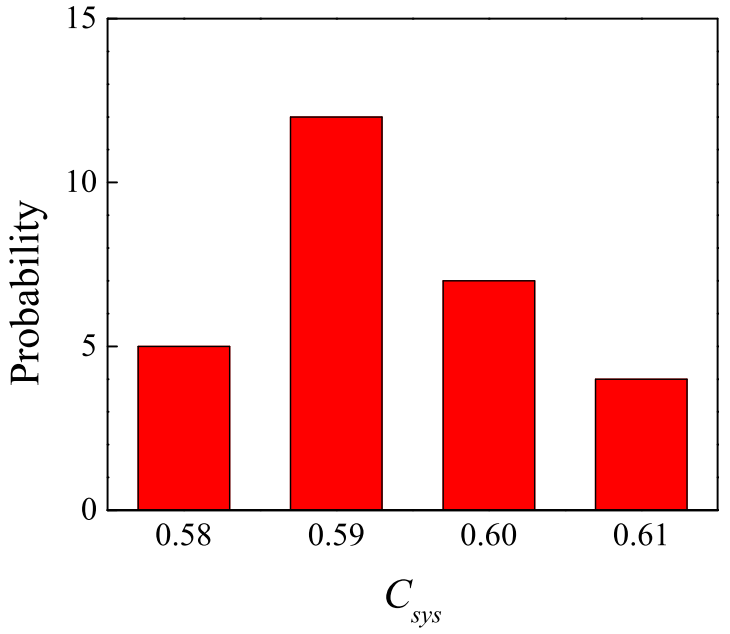

FIG. 8. Probability of $C_{\text {sys }}$.

respectively. The vector maps show a concentration (technically, a density) gradient in the central region, where the two streams mix. Areas with no vector indicate a constant concentration. Here, because we already know the bulk concentrations of the two streams, we can back-calculate the system parameter, $C_{\text {sys }}$. In the cases shown in Figs. 6 and 7, $C_{\text {sys }}$ was calculated as 0.59 . The experiment using 53-50 wt $\%$ mixing was repeated 13 times, whereas that of 53-51 wt \% mixing was repeated 15 times. The variation in the optimum $C_{\text {sys }}$ obtained from each experiment is shown in Fig. 8. Because 0.59 shows the largest probability, we determined the value of $C_{\text {sys }}$ to also be 0.59 . Note that the error in the concentration of zincate ions is less than $0.5 \%$ even when $C_{\mathrm{sys}}=0.60$ was employed.

\section{ZINC ANODE EXPERIMENTS}

\section{A. Experimental}

Figure 9 shows a schematic of the experiment setup. Metal zinc was used for both the anode (working electrode) and counter electrode. The width and depth of the electrode surface are 5 and $0.5 \mathrm{~mm}$, respectively. Zinc electrodeposits occur in the horizontal direction. Other surfaces exposed to the electrolyte were wrapped with Teflon tape to prevent the deposition. Before the experiments, the surface of the zinc anode was mechanically polished with \#1000 and \#2000 waterproof abrasive paper (FujiStar) for several minutes and then washed with deionized (DI) water to remove the residual abrasive medium. Zinc oxide powder of $99.5 \%$ purity (Kishida Kagaku) was initially dissolved into a $10 \mathrm{~mol} \mathrm{~L}^{-1}$ potassium

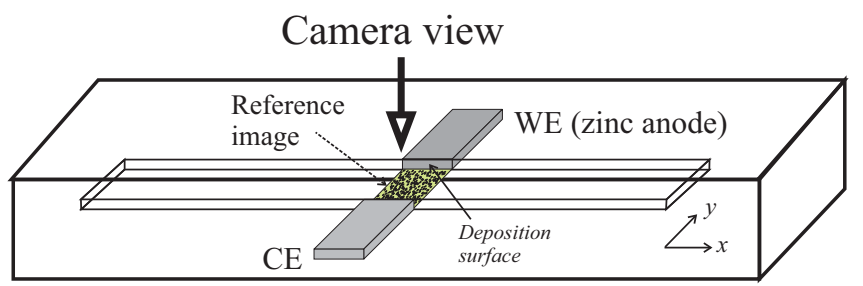

FIG. 9. Schematic of the battery cell.

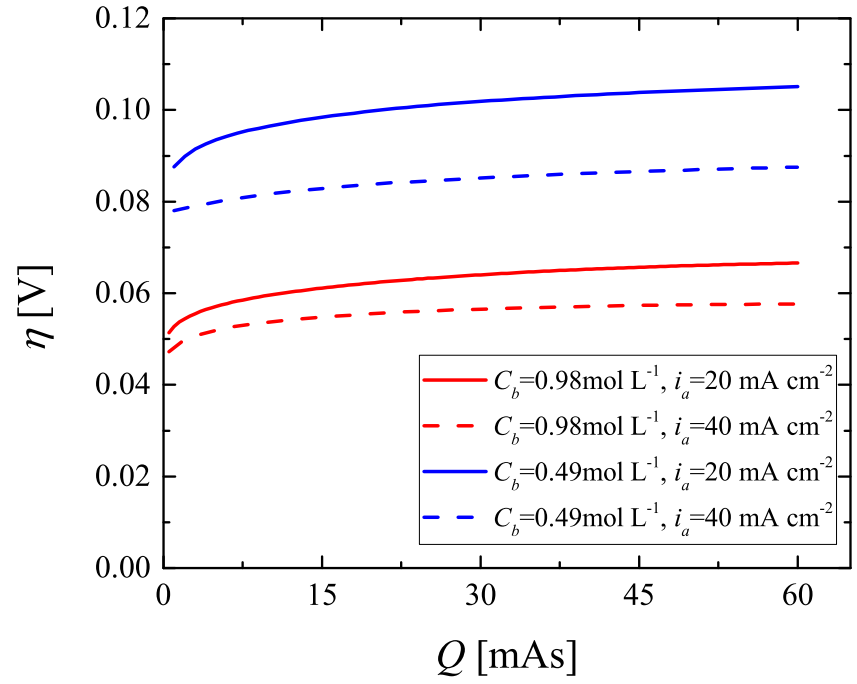

FIG. 10. Overpotential.

hydroxide $(\mathrm{KOH})$ solution as an electrolyte. The bulk zincate concentration, $C_{b}$, was set to 0.49 and $0.98 \mathrm{~mol} \mathrm{~L}^{-1}$.

The experiment setup and technique of the concentration measurement are basically the same as those used in the sugar water experiments. A part of the reference image and the zinc anode were captured from the top using an optical microscope. The imaging area was set to $x \times y=1.47 \times$ $1.10 \mathrm{~mm}$, and a central area of $0.68 \times 1.10 \mathrm{~mm}$ was analyzed. The image processing used to obtain the displacement vectors is conducted with an interrogation area of $32 \times 32$ pixels, resulting in a spatial resolution of the concentration of $18 \mu \mathrm{m}$.

Galvanostatic (constant current) charging experiments were conducted using an electrochemistry measurement system (Hokuto Denko, HZ-7000). The applied current density, $i_{a}$, was set to 20 and $40 \mathrm{~mA} \mathrm{~cm}^{-2}$. Under these conditions, the hydrogen evolution is negligibly small. The charging was terminated at a capacity of $Q=60 \mathrm{mAs}$ for all cases. The photo capture by a microscope was conducted every $15 \mathrm{mAs}$,

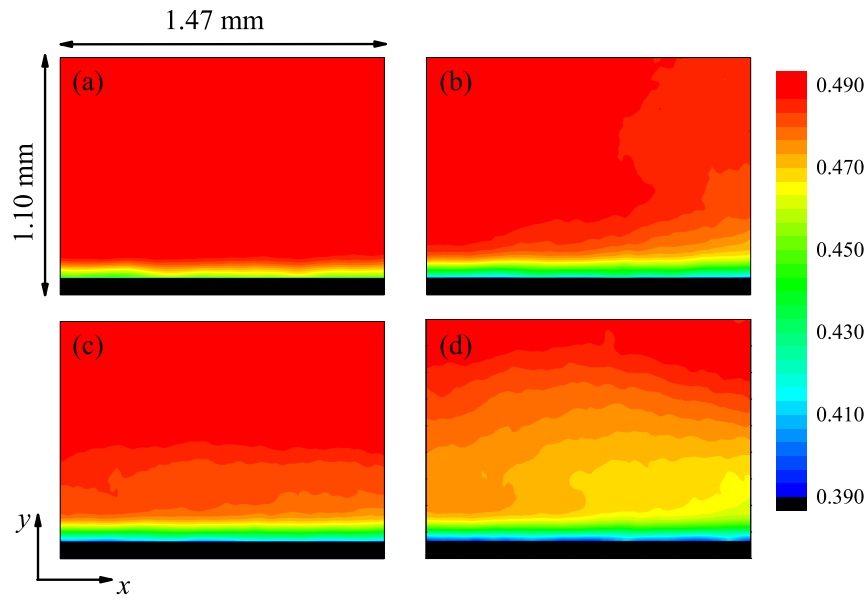

FIG. 11. An example of the time series changes of the zincate ion concentration distribution for $C_{b}=0.49 \mathrm{~mol} \mathrm{~L}^{-1}$ and $i_{a}=$ $20 \mathrm{~mA} \mathrm{~cm}^{-2}$ : (a) $Q=15 \mathrm{mAs}$, (b) $Q=30 \mathrm{mAs}$, (c) $Q=45 \mathrm{mAs}$, and (d) $Q=60 \mathrm{mAs}$. 


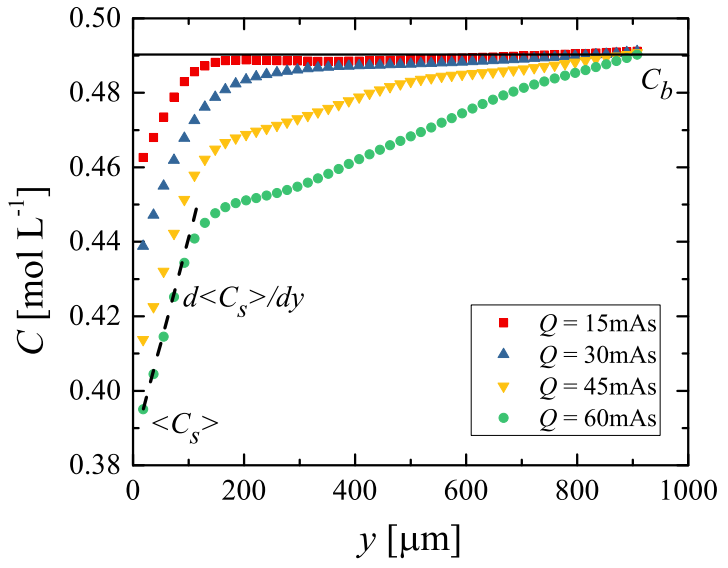

FIG. 12. Spatially averaged distribution of the zincate concentration against the distance from the anode surface.

i.e., every $30 \mathrm{~s}$ for $i_{a}=20 \mathrm{~mA} \mathrm{~cm}^{-2}$ and every $15 \mathrm{~s}$ for $i_{a}=40 \mathrm{~mA} \mathrm{~cm}^{-2}$. In addition, the overpotential of the zinc anode was measured using a $\mathrm{Hg} / \mathrm{HgO}$ reference electrode (BAS Inc., RE-61AP).

\section{B. Results and discussion}

\section{Overpotential}

An overpotential is a typical factor used to measure the distance from equilibrium. Figure 10 shows the overpotential of the zinc anode, $\eta$, against the charge capacity. The figure confirms that the overpotential is larger for a smaller $C_{b}$ and larger $i_{a}$, whereas the effect of the bulk concentration is relatively small.

\section{Concentration distribution of zincate ions}

Figure 11 shows an example of the time series changes of the zincate ion concentration distribution for $C_{b}=$ $0.49 \mathrm{~mol} \mathrm{~L}^{-1}$ and $i_{a}=20 \mathrm{~mA} \mathrm{~cm}^{-2}$. The black region

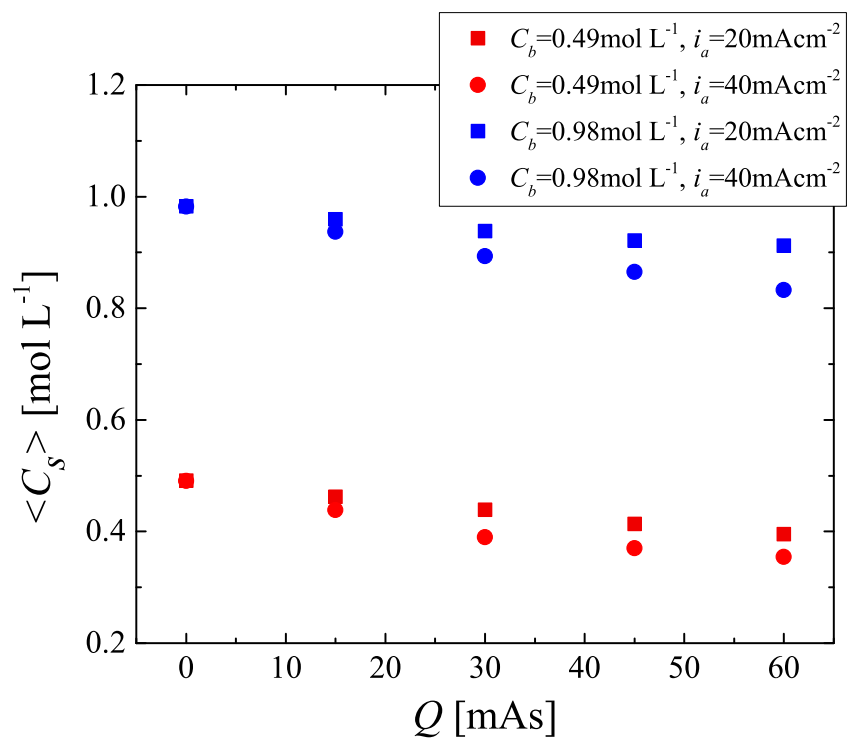

FIG. 13. Mean zincate ion concentration at the anode surface against the charge capacity.

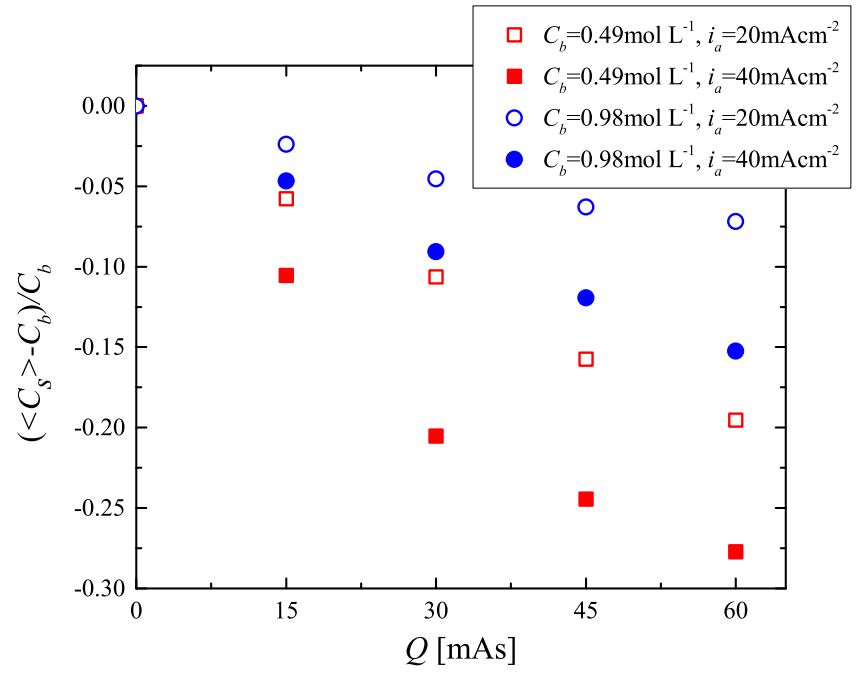

FIG. 14. Decrease in the surface concentration normalized using $C_{b}$ : (a) $i_{a}=20 \mathrm{~mA} \mathrm{~cm}^{-2}$ and (b) $i_{a}=40 \mathrm{~mA} \mathrm{~cm}^{-2}$.

represents a solid zinc anode and the red region corresponds to the bulk electrolyte. Note that zinc growth is barely observed and the shape is nearly flat because the charged capacity is small. This confirms the formation of a diffusion layer and that the zincate ion concentration decreases when approaching the electrode surface. Furthermore, the concentration on the anode surface decreases with the charging capacity.

To show the concentration distribution more clearly and qualitatively, Fig. 12 shows the zincate ion concentration against the distance from the anode surface. Here $y=0$ is the anode surface, and the concentration is spatially averaged in the $x$ direction. In addition, $C_{s}$ is the concentration at the surface, as discussed in the following.

Figure 13 shows the mean zincate ion concentration at the anode surface, $\left\langle C_{s}\right\rangle$, against the charge capacity. Here, $C_{s}$ denotes the concentration at the nearest point from the anode surface in the $y$ direction, and the brackets denote

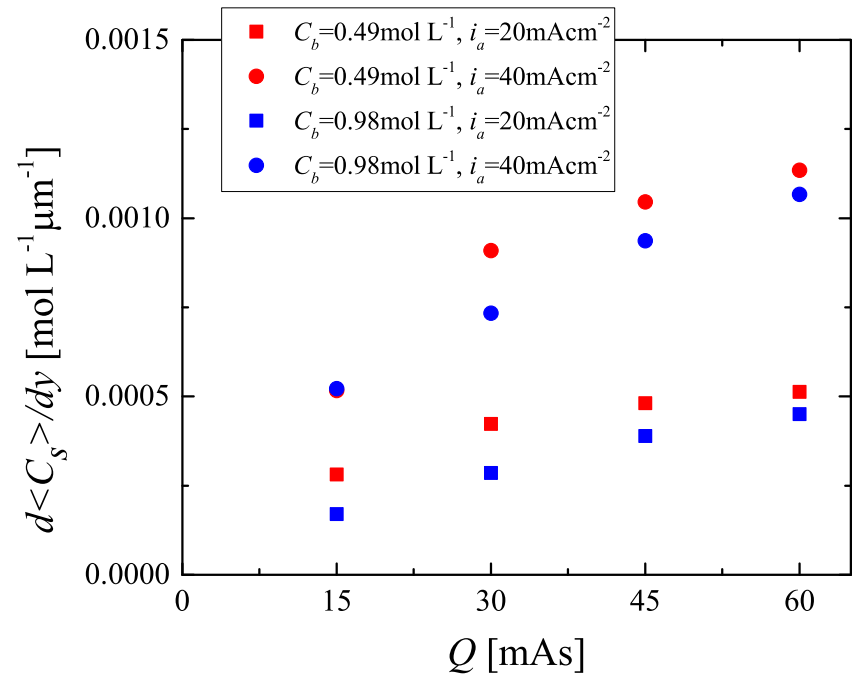

FIG. 15. Time series data of the concentration gradient on the anode surface 

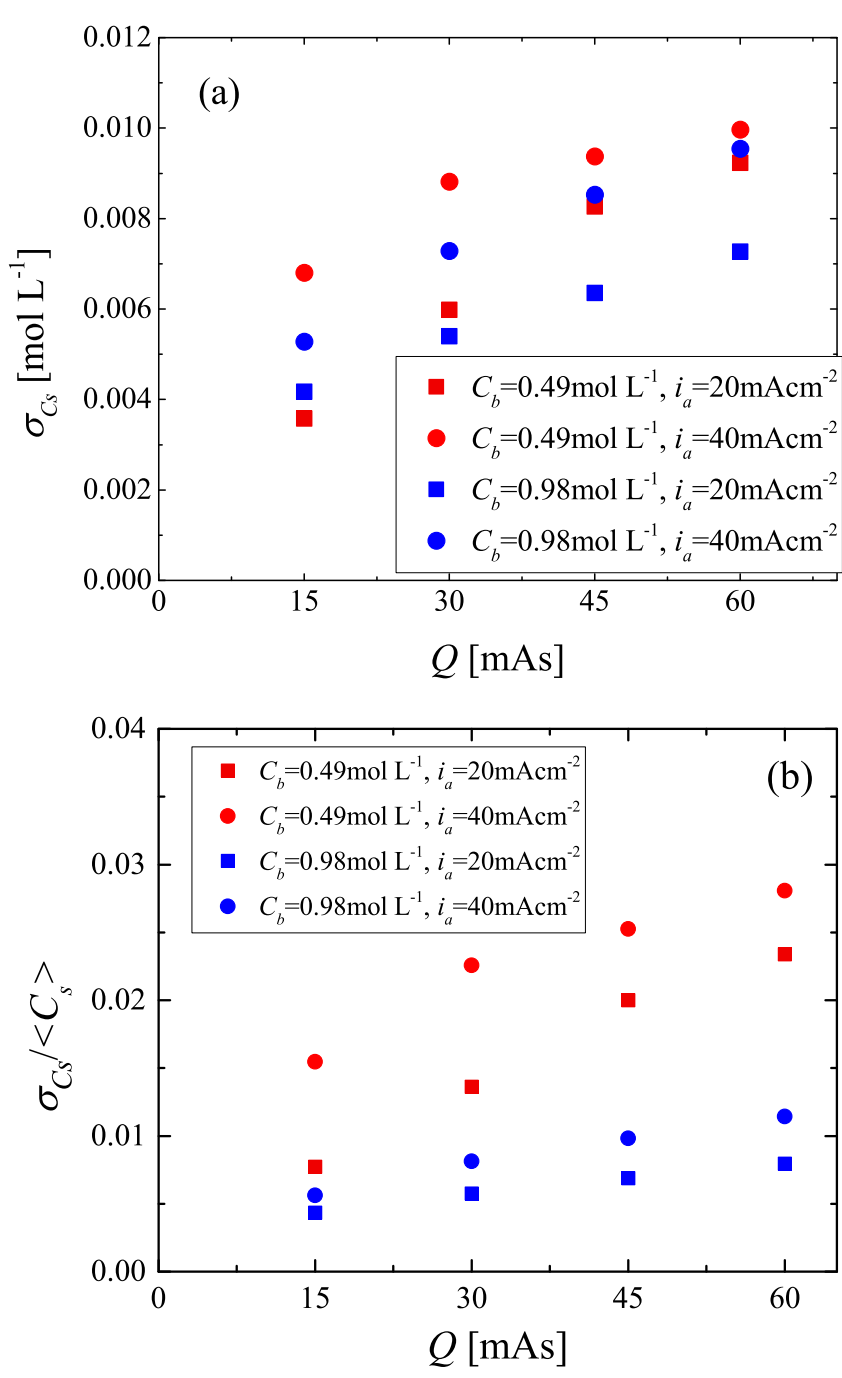

FIG. 16. Variance in zincate ion concentration along the anode surface: (a) $\sigma_{C_{s}}$ and (b) $\sigma_{C_{s}} /\left\langle\boldsymbol{C}_{s}\right\rangle$ against the charge capacity.

the average value along the surface. This confirms that the surface concentration becomes low with an increase in the charge capacity. It is also revealed that the decrease is larger for a larger $i_{a}$. Figures 14(a) and 14(b) show a decrease in the surface concentration when normalized using $C_{b}$ for $i_{a}=$ $20 \mathrm{~mA} \mathrm{~cm}^{-2}$ and $i_{a}=40 \mathrm{~mA} \mathrm{~cm}^{-2}$, respectively. The figures indicate that the relative decrease is larger for a smaller $C_{b}$.

Figure 15 shows the time-series data of the concentration gradient on the anode surface, $d\left\langle C_{s}\right\rangle / d y$, which is calculated using the five $\langle C\rangle$ points near the surface, as indicated in Fig. 12. Because the surface shape is nearly flat under the present conditions and the gradient in the $x$ direction is negligibly small, the gradient is taken only in the $y$ direction. The figure shows that the gradient gradually increases with the charge capacity and tends to level off. In addition, $d\left\langle C_{s}\right\rangle / d y$ is basically irrelevant to the bulk concentration but proportional to the applied current density.

Here, $d\left\langle C_{s}\right\rangle / d y$ is determined theoretically through the following equation:

$$
\frac{d\left\langle C_{s}\right\rangle}{d y}=\frac{i}{z F D},
$$

where $z, F$, and $D$ are the numbers of electrons of the zincate ions, Faraday's constant, and the molecular diffusion coefficient, respectively. The equation indicates that the concentration gradient is a function of only the applied current density, and $i$ is constant at $i=i_{a}$ under the present experiment conditions. Therefore, it is reasonable to find that the gradient is irrelevant to the bulk concentration but proportional to the applied current density. However, the figure shows that it takes time to reach a steady state. Although the reason for this is not clear, the surface condition such as a crystallographic orientation may have a significant effect initially. Furthermore, the molecular diffusion coefficient, $D$, was back calculated from the above equation, under the assumption that the concentration distribution near the electrode surface reached a steady state at $Q=60 \mathrm{mAs}$. The resulting values are $D=1.82-2.3010^{-9} \mathrm{~m}^{2} \mathrm{~s}^{-1}$. This is within the range of $1.6 \times 10^{-10}$ to $6 \times 10^{-9} \mathrm{~m}^{2} \mathrm{~s}^{-1}$, which is the range of the estimated molecular diffusion coefficient of zincate ions in $\mathrm{KOH}$ found in past studies [16,18]. These facts confirm the sufficient reliability of the BOS technique.

Figure 16 shows the variance in the zincate ion concentration along the anode surface, $\sigma_{C_{s}}$, and its normalized value by the mean surface concentration, $\left\langle C_{s}\right\rangle$, against the charge capacity. It illustrates that the variance in the concentration is larger for a larger $1_{a}$ and smaller $C_{b}$. In other words, it indicates experimentally that a nonuniform electrodeposition of zinc under far-equilibrium conditions is caused by an extremely large variance in the spatial concentration.

\section{CONCLUSIONS}

This study aimed at clarifying the zincate ion concentration distribution near the zinc anode upon charging. Galvanostatic charging experiments in a zinc battery cell were conducted. Images near the zinc anode were captured using an optical microscope to measure the zincate ion concentration distribution using the background-oriented Schlieren (BOS) technique. The main conclusions can be summarized as follows.

(1) The BOS technique enables us to quantitatively measure the zincate ion concentration near the zinc anode. The results are consistent with the theoretical expectation.

(2) The current density basically determines the concentration gradient at near the anode surface under the galvanostatic condition, but it takes time to reach a steady state.

(3) The spatial variance in the zincate ion concentration at the anode surface increases with the distance from equilibrium.

The present technique enables us to elucidate the mass transport of zincate ions in an electrolyte, which is considered one of the most important factors but was not easy to measure. We believe that this will accelerate the commercialization of zinc anode batteries. For example, it would be useful to determine the optimal concentration of additives needed to suppress zinc dendritic growth, where mass transport is often the key factor.

\section{ACKNOWLEDGMENTS}

The authors would like to thank Prof. Mitsuhiro Shikida (Hiroshima City University) and Prof. Hirotaka Hida (Kobe University) for preparing the reference images used for the 
BOS technique. We would also like to thank Genta Ukino (Nagoya University) for supporting the experiments and Prof.
Munekazu Motoyama (Nagoya University) for his useful comments.
[1] T. B. Reddy, Linden's Handbook of Batteries, 4th ed. (McGrawHill, New York, 2011).

[2] R. A. Huggins, Advanced Batteries (Springer, Berlin, 2010).

[3] Y. Ito, M. Nyce, R. Plivelich, M. Klien, D. Steingart, and S. Banerjee, Zinc morphology in zinc-nickel flow assisted batteries and impact on performance, J. Power Sources 196, 2340 (2011).

[4] Y. Ito, X. Wei, D. Desai, D. Steingart, and S. Banerjee, An indicator of zinc morphology transition in flowing alkaline electrolyte, J. Power Sources 211, 119 (2012).

[5] J. S. Newman and K. E. Thomas-Alyea, Electrochemical Systems, 3rd ed. (Wiley, New York, 2004).

[6] M. Wang and N. B. Ming, Concentration field oscillation in front of a dendrite tip in electrochemical deposition, Phys. Rev. A 45, 2493 (1992).

[7] J. Bockris, Z. Nagy, and D. Drazic, On the morphology of zinc electrodeposition from alkaline solutions, J. Electrochem. Soc. 120, 30 (1973).

[8] T. Mitsuhashi, Y. Ito, Y. Takeuchi, S. Harada, and T. Ujihara, Non-uniform electrodeposition of zinc on the (0001) oriented substrate, Thin Solid Films 590, 207 (2015).

[9] K. Ishikawa, Y. Ito, S. Harada, M. Tagawa, and T. Ujihara, Crystal orientation dependence of precipitate structure of electrodeposited Li metal on $\mathrm{Cu}$ current collectors, Cryst. Growth Des. 17, 2379 (2017).

[10] D. Grier, E. Ben-Jacob, R. Clarke, and L. M. Sander, Morphology and Microstructure in Electrochemical Deposition of Zinc, Phys. Rev. Lett. 56, 1264 (1986).

[11] P. P. Trigueros, J. Claret, F. Mas, and F. Sagués, Pattern morphologies in zinc electrodeposition, J. Electroanal Chem. 312, 219 (1991).

[12] Y. Cheng, H. Zhang, Q. Lai, X. Li, Q. Zheng, X. Xi, and C. Ding, Effect of temperature on the performances and in situ polarization analysis of zinc-nickel single flow batteries, J. Power Sources 249, 435 (2014).
[13] M. Gordon and G. Suppes, Convection battery-modeling, insight, and review, AIChE J. 59, 2833 (2013).

[14] K. I. Popov and N. V. Krstajić, The mechanism of spongy electrodeposits formation on inert substrate at low overpotentials, J. Appl. Electrochem. 13, 775 (1983).

[15] J. W. Diggle, A. R. Despic, and J. Bockris, The mechanism of the dendritic electrocrystallization of zinc, J. Electrochem. Soc. 116, 1503 (1969).

[16] J. Riede, T. Turek, and U. Kunz, Critical zinc ion concentration on the electrode surface determines dendritic zinc growth during charging a zinc air battery, Electrochim. Acta 269, 217 (2018).

[17] J. Dundáleka, I. Šnajdra, O. Libánskýa, J. Vránaa, J. Pocedičb, P. Mazúrb, and J. Koseka, Zinc electrodeposition from flowing alkaline zincate solutions: Role of hydrogen evolution reaction, J. Power Sources 372, 221 (2017).

[18] M. Saleh, J. Weidner, and B. Ateya, Electrowinning of nonnoble metals with simultaneous hydrogen evolution at flowthrough porous electrodes: I. Theoretical, J. Electrochem. Soc. 142, 4113 (1995).

[19] J. K. Weaver, F. R. McLarnon, and E. J. Cairns, Experimental and theoretical study of concentration distributions in a model pore electrode. I. Measurement of two-dimensional concentration gradients in a zinc model pore, J. Electrochem. Soc. 138, 2572 (1991).

[20] M. J. Hargather and G. S. Settles, A comparison of three quantitative Schlieren techniques, Opt. Lasers Eng. 50, 8 (2012).

[21] H. Richard and M. Raffel, Principle and applications of the background oriented Schlieren (BOS) method, Meas. Sci. Technol. 12, 1576 (2001).

[22] V. Venkatakrishnan and G. E. A. Meier, Density measurements using the background oriented Schlieren technique, Exp. Fluids 37, 237 (2004).

[23] M. Raffel, Background-oriented Schlieren (BOS) technique, Exp. Fluids 56, 60 (2015). 